\title{
Developing creativity in higher education for 21st century learners: A protocol for a scoping review
}

\author{
Arlene Egan $^{\mathrm{a}, *}$, Rebecca Maguire ${ }^{\mathrm{a}}$, Lauren Christophers ${ }^{\mathrm{b}}$, Brendan Rooney ${ }^{\mathrm{b}}$ \\ a CRILT, National College of Ireland, IFSC, Dublin 1, Ireland \\ ${ }^{\mathrm{b}}$ School of Psychology, UCD, Belfield, Dublin 4, Ireland
}

\section{A R T I C L E I N F O}

\section{Article history:}

Received 13 July 2016

Received in revised form 13 December 2016

Accepted 28 December 2016

Available online 17 January 2017

\section{Keywords:}

Creativity

Higher education

Scoping review

Framework

Development

\begin{abstract}
A B S T R A C T
There is an abundance of literature highlighting the need to focus on enhancing students' creativity in higher education. However, currently there is a gap in awareness of evidencebased initiatives being employed in institutions to address this need. The debate on how to best characterise creativity has not yet reach consensus therefore, we present a protocol for a new review that will identify the characteristics of the frameworks as well as the tools being used by educators to formally develop students' creativity in higher education. It will also provide insight into how these educators are defining creativity. This knowledge will enhance understanding of how creativity, a necessary skill for 21st century learners is being harvested, valued, and described in higher education.
\end{abstract}

(c) 2017 Elsevier Ltd. All rights reserved.

\section{Introduction}

Creativity has been regarded as one of the crucial skills in the toolkit of the 21st century learner and indeed key to effective learning in higher education and beyond (Jahnke, Haertel, \& Wildt, 2015; Nissim, Weissblueth, Scott-Webber, \& Amar, 2016; Rampersad \& Patel, 2014). It has even been described as 'the cultural capital of the twenty-first century' (Sheridan-Rabideau, 2010, p. 54). While creativity has been noted as a significant skill across the life-span, Livingston (2010) reported that creativity is an essential skill for students to harvest in higher education as it has a direct link to the development of "content knowledge and skills in a culture infused at new levels by investigation, cooperation, connection, integration and synthesis" (p. 59). Given the high level of agreement on its importance, the question remains; what are higher education institutions, and the educators within doing to foster, nurture and develop student's levels of creativity. On one hand, there is the positive idea stated by Tosey (2006), who commented that higher education institutions cannot avoid creativity, as they harbour individuals 'who are constantly using their ingenuity in interaction with others' (p. 33). There is also evidence that creativity is a specific requirement in terms of day-to-day teaching practice where the intention of learning is to;

"generate ideas and possibilities, invent ways of exploring problems, complex situations and systems [or] combine ideas and things in novel ways" (Jackson \& Shaw, 2005, p.105).

\footnotetext{
* Corresponding author.

E-mail addresses: arlene.egan@ncirl.ie (A. Egan), Rebecca.maguire@ncirl.ie (R. Maguire), laurenchristophers@gmail.com (L. Christophers), Brendan.rooney@ucd.ie (B. Rooney).
} 
These ideas imply that creativity could be considered part of the fabric of higher education. However, the challenges associated with enhancing students' creativity in higher educaton have been widely acknowledged in the literature. These range from the rigid management practices in higher education which stiffle creativity (MacLaren, 2012) to designing assessment strategies that can relaibly assess creativity (Cowan, 2006). Of course decisions concerning assessment depend primarily on whether the educator takes the position that creativity is fundamentally product oriented (Amabile, 1983; Elliott, 1995) or process oriented (Johnson-Laird, 1987) and lack of consenuss on this matter adds to the challenge. Kleiman (2008, p. 212) has commented that if experiences of teaching creativity could be placed on one continuum, fulfilment-focus would sit at the upper end while constraint-focused would sit at the lower, which reflects the negative perceptions of some academics teaching creativity in higher education. This negativity has been associated with the fact that creativity and the creative processes can challenge established teaching and learning strategies, as well as the institutional ethos.

\subsection{Understanding creativity}

Creativity has long been a topic of interest within psychology and its cognate disciplines, with particular attention paid to this concept in the latter half of the twentieth century, especially in relation generating theories of creativity (for example; Kris, 1952; Mendelsohn, 1976; Mendick, 1962). Today, the term creativity is employed in many different contexts with an increasing recognition of its value in numerous domains including technology, business and entrepreneurship (e.g., Cooper, 2000; Zimmerer \& Scarborough, 2008). This emphasis has been extended to higher education with many studies recognising its value within third level institutions (Garrison \& Kanuka, 2004). Contemporary work is regularly championing creativity, citing it as a crucial skill for the 21 st century (Greiff, Niepel, \& Wustenberg, 2015). Links have been made between levels of creativity and other valued skills including critical thinking (Dwyer, Hogan, \& Stewart, 2014), as well as communication and innovation (Kratzer et al., 2004).

However, since the turn of the 21st century, creativity has become more pervasive as a construct, there are many inconsistencies in the definitions adopted (Batey, 2012; Meusburger, Funke, \& Wunder, 2009). Creativity has been described as 'maligned, neglected and misunderstood' (Azzam, 2009, p. 22). This is reflected in a diversity of intuitive conceptions of creativity, with individuals adopting different views as to what is meant by creativity (Gluck, Ernst, \& Unger, 2002). Within higher education, much debate has surrounded whether creativity can be taught (Livingston, 2010; Jackson, Oliver, Shaw, \& Wisdom, 2006). Smith (2006) suggests that while society has a view of creativity as a talent, shown by some, other research is suggesting that creativity is perhaps developed through a teachable process and

‘. . colleges and universities can work to create curricula, pedagogies, cocurricular programming and a general institutional environment to support creative development' (p. 24).

The lack of agreement on whether creativity can be taught or not shows confusion in the field which is compounded by the lack of consensus of what creativity involves and how this might be influenced by contextual factors.

Attempts have been made to establish what academics themselves understand as creativity. For example Jackson and Shaw (2005) report certain themes in definitions including originality, innovation, transfer and adaptation of ideas. Using qualitative analysis Kleiman (2008) observed five categories of views of the creative experience: that which is constraint focused, process focused, product focused, transformation focused and fulfilment focussed. Rather than trying to reach consensus on how to define creativity, it may be more advantageous to examine themes or characteristics associated with how it is understood in different contexts. Efforts to identifying approaches that will enhance student's levels of creativity have been made (e.g., Jeffrey \& Craft, 2004, Loveless, Burton, \& Turvey, 2006) however, as of yet there has been no one method espoused for its effectiveness in increasing students' levels of creativity in higher education. This again relates to the idea that creativity practiced in higher education is deeply situated. Given the contextual and ecological influences on teacher's approaches, strategies, environment and thinking, identifying principles to aid effective design would be considered a worthwhile exercise.

\subsection{Why conduct a review on creativity}

Through the review, frameworks being used by educators and practitioners in higher education will be identified, examined and deconstructed in order to generate a clearer understanding of those tools, frameworks and their associated characteristics. Loveless et al. (2006, p. 4) refer to Craft (2000) in their description of a useful theoretical framework for recognising and developing creativity as 'an interaction between characteristics in people and communities, creative processes, subject domains and wider social and cultural contexts'. By examining these frameworks we can inform evidencebased practice in higher education. Scoping studies are used extensively in health research to build bodies of knowledge based on practice and evidence. Education should follow suit, as these types of studies can have implications for practice at individual, institutional and global levels.

The increase in multidisciplinary approaches and methodologies stemming from cognitive science can shed new light on the meaning of creativity which is why an analysis of contemporary research is merited. Gaspar and Mabic (2015, p. 599600 ) remind us of the prominence of creativity in the majority of European strategic reports on higher education, including; EUROPE 2020 (European Commission, 2010). Publications Office of the European Union. and), EUA (2011). Smart People for 
Smart Growth and the Lisbon Declaration (2007) - Europe's Universities Beyond 2010, and yet the stark reality remains that, as educators, there is very little information on best practice in existence.

There have been a small number of reviews that have been conducted that expressly focus on developing students' creativity, however, these have been carried out in particular subject areas; for example, nursing (see Chan, 2013; Duhamel, 2016) or music education (Running, 2008). Following his review, Running (2008) suggested that further investigations are warranted to ascertain whether creativity can be taught or not at higher education. It would appear that questions associated with definition (or description) and effective approaches to teaching creativity have been asked for decades. Currently, no review exists that can offer insight into initiatives that have been employed across a range of subject settings. Conducting a scoping review will contribute to a greater understanding of the characteristics of creativity in practice across different contexts in higher education. It could also be used to inform further knowledge gathering in relation to pedagogic practice, for example by means of larger-scale, quantitative investigations. Building on the protocol analysis in this way would lead to an enhanced understanding of practices of creativity in a broader range of educational contexts, beyond an analysis of published literature.

Through an understanding of the frameworks employed and associated definitions, it is possible to gain insight into how creativity is being thought about and taught as a 21 st century skill in higher education.

\subsection{Review objectives}

The primary objective of this review is to establish how creativity is being formally developed in higher education in order to inform practice by (1) identifying the frameworks and tools being used by educators to explicitly develop student's levels of creativity (2) identifying the characteristics of creativity that these educators associate with their frameworks.

Table 1

Six stages for a scoping study (Arksey \& O'Malley, 2005) with recommendations from Daudt et al. (2013).

\begin{tabular}{|c|c|}
\hline Stage & Recommendation \\
\hline $\begin{array}{l}\text { 1.Identifying the } \\
\text { research question }\end{array}$ & $\begin{array}{l}\text { 1. Conduct considerable research about scoping studies to ensure an appropriate match between the scoping } \\
\text { methodology and the research interest. Consider the methodology's objectives, boundaries, and the types of } \\
\text { research that it can best support. } \\
\text { 2. Link the purpose of the research with the research question and attend to suggestions to clarify concepts } \\
\text { within the research question. }\end{array}$ \\
\hline 2. Identifying relevant studies & $\begin{array}{l}\text { 1. Remain flexible to revise the research question and/or search terms. } \\
\text { 2. Build both a multidisciplinary and inter-professional team. Include someone experienced with scoping } \\
\text { studies and suitable stakeholders if possible. } \\
\text { 3. Choose a small suitable group from the larger research team of qualified researchers and professionals with } \\
\text { enough breadth of expertise for this stage to ensure timely completion of the study }\end{array}$ \\
\hline 3. Study selection & $\begin{array}{l}\text { 1. For large research teams, take a three-tiered approach to study selection. Divide entire team into smaller } \\
\text { teams with responsibility for equal portions of the selected studies. Ask each person to review his/her selected } \\
\text { studies for inclusion or exclusion. Have each small team compare its results. If disagreement, involve a third } \\
\text { reviewer. } \\
\text { 2. Assess the quality of studies to be either included or excluded for charting. Quality can be assessed using } \\
\text { validated instruments }\end{array}$ \\
\hline 4. Charting the data & $\begin{array}{l}\text { 1. Conduct a trial charting exercise and group consultation to determine if adjustments should be made to the } \\
\text { chart (variables being measured) and to ensure that the research team is charting consistently. } \\
\text { 2. Create a comprehensive chart, involving both high-level data and micro-level data, in order to capture a rich } \\
\text { set of data. } \\
\text { 3. Hold frequent meetings to ensure effective communication about consistent charting. Hold additional longer } \\
\text { meetings when necessary. } \\
\text { 4. For large research teams, take a three-tiered approach to charting the data. Divide entire team into smaller } \\
\text { teams with responsibility for equal portions of the selected studies. Pick different team members from stage \#3. } \\
\text { Ask each person to review his/her selected studies for inclusion or exclusion. Have each small team compare its } \\
\text { results. Have one independent reviewer read and chart all studies. Have independent reviewer compare his/her } \\
\text { charting with the charting of all other team members. Discuss any discrepancies. } \\
\text { 5. Improve data management by assigning each study a unique identifying number to avoid confusion. }\end{array}$ \\
\hline $\begin{array}{l}\text { 5. Collating, summarising and } \\
\text { reporting the results }\end{array}$ & $\begin{array}{l}\text { 1. Engage a small working group from the larger team to make meaning out of the data and to make choices } \\
\text { about the data on which to focus. }\end{array}$ \\
\hline 6. Consultation exercise & $\begin{array}{l}\text { 1. If there are stakeholders (e.g., policy makers and allied researchers) who were not part of your research team, } \\
\text { engage in a consultation process with them. Consult stakeholders only if the actual scoping study results are } \\
\text { germane. } \\
\text { 2. Recognize that the inability to share a scoping study's findings with stakeholders may be an indication that } \\
\text { future research must be done beyond the scoping study in order to make a meaningful contribution to } \\
\text { professional practice. } \\
\text { 3. Systematic engagement with practitioners in established networks of interest, such as, JISC mail Imaginative } \\
\text { Curriculum Network or the Creative Network. }\end{array}$ \\
\hline
\end{tabular}




\section{Methods}

\subsection{Study design}

The study design will entail a scoping review rather than a systematic review. This form of review has been described as increasingly popular for synthesising research evidence (e.g., Levac, Coloquhoun, \& Brien, 2010; Daudt, van Mossel, \& Scott, 2013). A scoping review has been selected owing to the breath of the research objectives underpinning the present investigation. As distinct from a systematic review, the aim of a scoping review is to map "key concepts, types of evidence, and gaps in the research related to a defined area or field by systematically searching, selecting, and synthesising existing knowledge" (Colquhoun et al., 2014, p. 1294). As such scoping reviews tend to focus on the breath rather than the depth of evidence (Prihodova, Guerin, \& Kernohan, 2015). One formal approach to guide a scoping review for broad research questions has been the six stage framework originally proposed by Arksey and O'Malley (2005) and developed by Levac et al. (2010) and Daudt et al. (2013) (see Table 1). Davis et al., (2009), have described this framework as 'an interpretative scoping literature review methodology' (p.1388). These researchers explain that this method is effective for use when the review will focus on studies that have disparity in their findings. In advancing the efficacy of the framework, Levac et al. (2010) provided comprehensive additions to the scoping framework by retaining the main headings suggested by Arksey and O'Malley (2005), and providing a more detailed guidance for researchers on how to operate at each stage. This work was advanced even further by Daudt et al. (2013) who proposed further recommendations, outlined in Table 1 below, which will be used for guiding this scoping review.

Originally, Stage 6 was suggested to be an optional stage, whereby experts in areas connected with the research question are invited to review and comment on the stages of the study to ensure it is being effectively executed and progressing in an un-biased way. Both Levac et al. (2010) and Daudt et al. (2013) highly recommend that this stage be included in the process and it will be retained for the present review.

\subsection{Search strategy and source selection}

A search strategy was designed in collaboration with an information search expert from the college library. There are parameters established for the study that will influence the extent of the search. Specifically; only studies published since 2000 will be considered based on the connection between creativity and 21 st century learning. Also, only studies available in English will be considered, (as that is the only language of the researchers) and only studies in peer-reviewed journals will be considered. A systematic search will be conducted on the following electronic collections and databases; EBSCOhost Psychology and Behavioural Sciences Collection, ScienceDirect, Education Research Complete and Web of Science (Science and Social Science Index). Searches of Titles, abstracts and keywords will be conducted by AE and RM using the types of search terms indicated in Table 2.

\subsubsection{Study inclusion and exclusion criteria}

Given the breath of the search terms outlined above, we expect that a broad range of papers will result. Aside from the parameters outlined above other inclusion criteria have also been identified. As the primary objectives of this scoping review concerns the identification of frameworks for creativity enhancement and their associated characteristics, both qualitative and quantitative research will be considered. The research must have been conducted in a higher education context with students either as part of a module or as a standalone module. We are not excluding studies based on specific subject area. Finally, to be considered eligible, the papers under review must state in their own words that the aim of the research was to enhance students' levels of creativity.

\subsubsection{Article selection process}

The team of researchers have been identified and have expertise in relation to searching ability and methodological understanding. In line with the recommendations of Daudt et al. (2013), the researchers will work through the six stage framework outlined earlier in Table 1. In order to ensure that the framework for reviewing is being used appropriately, we will also ensure regular structured meetings are scheduled to maintain a clear focus on the aims and objectives of the review and to provide an open forum to allow for ongoing dialogue between the team to ensure that researchers maintain focus in

Table 2

Sample of search terms for the ERIC database.

Step Search terms

1 To identify relevant research through title and all text in order to generate a wide range of responses

creat*(framework OR tool AND develop* OR college OR higher education OR third level OR university OR tertiary)

2 creat*(approach* OR initiative AND develop* OR enhance OR increase OR college OR higher education OR third level OR university OR tertiary)

3 To identify relevant research through title and abstract in order to refine range of responses

creat*(framework OR tool OR approach* OR initiative AND develop* OR enhance* OR increas* OR college OR higher education OR third level OR university OR tertiary) 
terms of the evidence and data they collect. It will be necessary for the researchers to have an ability to identify frameworks which have implicit characteristics as well as those that have been explicitly developed, therefore the need to support ongoing dialogue is envisaged. This further supports our use of the six stage framework (Arksey \& O’Malley, 2005) as rather that forcing researchers to operate rigidly, 'it is based on an iterative, conceptual and interpretative approach' (Davis, Drey, \& Gould, 2009; p. 1388). An extensive search of databases will be conducted in order to identify all eligible studies. The reference sections of all eligible studies will also be searched to identify any other literature not sourced through the online search strategy.

\subsection{Data extraction and synthesis}

Studies will be identified initially by screening the Titles and abstracts and in circumstances where it is not possible to make a decision based on the title and/or abstract, the full text will be reviewed. It is expected that searching will be iterative and checks will be built into the process to ensure that the inclusion/exclusion criteria are being implemented consistently. One such check will be to have the allocated researchers review a sample of their selection and to follow this with a wider team meeting to ascertain the appropriateness of the studies. Once the results of the search are imported into EndNote, duplicates will be removed. The remaining studies will be independently reviewed by two researchers to ensure they meet the inclusion criteria, which is a valuable step in the process highlighted by Arksey and O'Malley (2005). Once these researchers have reviewed the papers and have made initial decisions in relation to inclusion and exclusion (or studies to be further reviewed) they will meet to discuss their decisions. At this point, a third researcher will arbitrate any lack of agreement or outstanding decisions.

\subsubsection{Charting}

The recommendations of both Arksey and O'Malley (2005) and Levac et al. (2010) and Daudt et al. (2013) recommend charting the data using a charting form. In their paper Arksey and O'Malley (2005) compare this exercise to a 'narrative review'. From the perspective of a scoping review, the main purpose of charting is to develop consensus on what information should be extracted from each study. These researchers offer a broad framework for charting which will be adopted for this scoping review. Specifically, they suggest that information should be recorded on;

- Author(s), year of publication, study location

- Intervention type, and comparator (if any); duration of the intervention

- Study populations

- Aims of the study

- Methodology

- Outcome measures

- Important results

(p. $16-17$ )

Given the specific outcomes of the present scoping review, there will be adaptations made to this chart to allow characteristics of creativity and the elements of the framework and tools used to be captured. We are also very mindful of the influence of context and ecological dimensions of the process by which creativity is considered in different educational practices. Where possible, an examination of contextual dimensions of the studies will be recorded. In line with further recommendations offered by Daudt et al. (2013) two researchers will use this form to extract data from the first 7 studies, as a means of a trial charting exrcise. This enables an early check to be performed on consistency and relevance. Once this has been established and any changes considered, the remaining researchers will commence data extraction.

Given the likelihood that both qualitative and quantitative data will emerge from this study, data synthesis and analysis will be conducted using both 'a descriptive numerical summary and a thematic analysis' (Levac et al., 2010; p. 6). The results of this process will be used to inform the consultation process which is likely to include stakeholders from higher education, industry and researchers in the area of creativity.

Before the final write-up, we will conduct a consultation exercise which is designed to gather the views and opinions of stakeholders that are interested in the topic, but are not directly involved as a member of the research team. By incorporating this stage, we are provided with alternative perspectives on our approach and findings, which will add value to our review. The consultation team that envisage will consist of two members of the academic community who teach in higher education contexts, along with a recognised researcher in this area. We also feel it is useful to incorporate the views from stakeholders outside of the academic community. Therefore we will also invite members of the business community to be part of the consultation panel. These representatives have been identified as working in business domains that are considered 'creative' and 'innovative'.

\section{Discussion}

Creativity is being touted as a vital skill for the 21st century. However, as of yet, we have not reached agreement on key aspects of creativity development in higher education. In fact the same questions that have been asked in the past are being 
Table 3

Proposed timeline for scoping review completion.

\begin{tabular}{llll}
\hline Stage & Task & Time & Owner(s) \\
\hline 1 & Identifying the research question and writing the protocol & Months 1 -2 & AE, RM, BR \\
2 & Identifying relevant studies & Month 3 & AE, RM \\
3 & Study selection & Months $4-6$ & BR, LC \\
4 & Charting the data & Month 7 & AE, RM, LC \\
5 & Collating, summarising and reporting the results & Months $8-10$ & AE, RM, BR \\
6 & Consultation exercise & Months 10 - 12 & Industry, academic and research experts \\
& Write-up & Months 12 -14 & AE, RM, BR \\
\hline
\end{tabular}

asked again today; 'Is creativity a gift, or can it be developed? Can it be learned? Can it be taught?' Running (2008). As there is a lack of consensus on the characteristics of creativity or the most effective ways to improve it, this protocol has been developed following many discussions and debates with academics and researchers in relation to the efficacy, relevance and value of creativity to education. The results from this review will identify how educators have been thinking about, talking about and focusing on the development of students' creativity.

\section{Proposed timeline and researcher involvement}

It is anticipated that this scoping study will be completed within 14 months, the schedule of activities is outlined in Table 3 below.

\section{Conflict of interest}

None.

\section{Funding}

This research did not receive any specific grant from funding agencies in the public, commercial, or non-for-profit sectors.

\section{References}

Amabile, T. M. (1983). The social psychology of creativity. New York: Springer-Verlag.

Arksey, H., \& O’Malley, L. (2005). Scoping studies: Towards a methodological framework. International Journal of Social Research Methodology, 8(1), 19-32. Azzam, A. (2009). Why creativity now? a conversation with sir ken robinson. Educational Leadership, 67(1), 22-26.

Batey, M. (2012). The measurement of creativity: From definitional consensus to the introduction of a new heuristic framework. Creativity Research Journal, 24(1), 55-65.

Chan, Z. C. Y. (2013). A systematic review of creative thinking/creativity in nursing education? Nurse Education Today, 33(11), 1382-1387.

Colquhoun, H., Levac, D., O'Brien, S., Tricco, A. C., Perrier, L., Kastner, M., et al. (2014). Scoping reviews: Time for clarity in definition, methods, and reporting. Journal of Clinical Epidemology, 67(12), 1291-1294.

Cooper, R. (2000). Information Technology Development Creativity: A case study of attempted radical change. MIS Quarterly, 24(2), $245-276$.

Cowan, J. (2006). How should I assess creativity? In N. Jackson, M. Oliver, M. Shaw, \& J. Wisdom (Eds.), Developing creativity in higher education: An imaginative curriculum (pp. 156-172).New York: Routledge.

Craft, A. (2000). Creativity across the primary curriculum: Framing and developing practice. London: Routledge.

Daudt, H., van Mossel, C., \& Scott, S. J. (2013). Enhancing the scoping study methodology: A large intre-professional team's experience with Arskey and O'Malley's framework. BMC Med Research, 13(1), 48-56.

Davis, K., Drey, N., \& Gould, D. (2009). What are scoping studies: A review of the nursing literature. International Journal of Nursing Studies, 46, 1386-1400.

Duhamel, K. V. (2016). Bringing us back to our creative senses: Fostering creativity in graduate-level nursing education: A literature review. Nurse Education Today, 45, 51-54

Dwyer, C. P., Hogan, M. J., \& Stewart, I. (2014). An integrated critical thinking framework for the 21 st century. Thinking Skills and Creativity, 12, 43-52. European University Association (2011). Smart people for smart growth. Belgium: European University Association.

European Commission (2010). Europe 2020: A Strategy for Smart, Sustainable and Inclusive Growth: Communication from the Commission. Publications Office of the European Union.

Elliott, D. (1995). Music matters: A new philosophy of music education. New York: Oxford University Press.

Garrison, R., \& Kanuka, H. (2004). Blended learning: Uncovering its transformative potential in higher education? Internet and Higher Education, 7(2), 95105.

Gaspar, D., \& Mabic, M. (2015). Creativity in higher education. Universal Journal of Educational Research, 5(1 S1), 598-605.

Gluck, J., Ernst, R., \& Unger, F. (2002). How creativies define creativity: Definitions reflect different types of creativity. Communication Research Journal, 14(1), 55-67.

Greiff, S., Niepel, C., \& Wustenberg, S. (2015). 21 st century skills: Recent advancements and international developments. Special Issue. Thinking Skills and Creativity, 8, 1-3.

Jackson, N., \& Shaw, M. (2005). Subject perspectives on creativity: A preliminary synthesis. Imaginative curriculum symposium on creativity in higher education at the higher education academy annual conference, [Online at http://www.heaacademy.ac.uk/2762.htm].

Jackson, N., Oliver, M., Shaw, M., \& Wisdom, J. (2006). Developing creativity in higher education: An imaginative curriculum. New York: Routledge.

Jahnke, I., Haertel, T., \& Wildt, J. (2015). Teachers' conceptions of student creatvity in higher education. Innovations in Education and Teaching International, 52, $1-9$.

Jeffrey, B., \& Craft, A. (2004). Teaching creatively and teaching for creativity: Distinctions and relationships. Educational Studies, $30(1), 77-87$.

Johnson-Laird, P. N. (1987). Reasoning: Imagining and creating. Bulletin of the Council for Research in Music Education, $95,71-87$.

Kleiman, P. (2008). Towards transformation: Conceptions of creativity in higher education. Innovations in Education and Teaching International, 45(3), 209217. 
Kratzer, Jan, Leenders, Oger Th A. J., \& van Engelen, Jo M. L. (2004). Stimulating the potential: Creative performance and communication in innovation teams. Creativity and Innovation Management, 13, 63-71.

Kris, E. (1952). Psychoanalytic explorations in art. New York: International Universities Press.

Levac, D., Coloquhoun, H., \& Brien, O. (2010). Scoping studies: Advancing the methodology. Implementation Science, 5(1), 69-77.

Livingston, L. (2010). Teaching creativity in higher education. Arts Education Policy and Review, 111, 59-62.

Loveless, A., Burton, J., \& Turvey, K. (2006). Developing conceptual frameworks for creativity: ICT and teacher education. Thinking Skills and Creativity, 1(1), 313.

MacLaren, I. (2012). The contradictions of policy and practice: Creativity in higher education. London Review of Education, 10(2), 159-172.

Mendelsohn, G. (1976). Associative and attentional processes in creative performance. Journal of Personality, 44, 341-369.

Mendick, S. (1962). The associative basis of the creative process. Psychology Review, 69, 220-232.

Meusburger, P., Funke, J., \& Wunder, E. (2009). Milieus of Creativity: An interdisciplinary approach to spatiality of creativity. Springer Science and Business Media B.V..

Nissim, Y., Weissblueth, E., Scott-Webber, L., \& Amar, S. (2016). The effect of a stimulating learning environment on pre-service teachers' motivation and 21 st century skills. Journal of Education and Learning, 5(3), 29-39.

Prihodova, L., Guerin, S., \& Kernohan, G. (2015). Knowledge transfer and exchange frameworks in health and their applicability to palliative care: Scoping review protocol. Journal of Advanced Nursing, 71(7), 1717-1725.

Rampersad, G., \& Patel, F. (2014). Creativity as a desirable graduate attribute: Implications for curriculum design and employability. Asia-Pacific Journal of Cooperative Education, 15(1), 1-11.

Running, D. J. (2008). Creativity research in music education a review (1980-2005). Update: Applications of Research in Music Education, $27(1)$, 41-48.

Sheridan-Rabideau, M. (2010). Creativity repositioned. Arts Education Policy Review, 111, 54-58.

Smith, T. (2006). Creativity research review: Some lessons for higher education. Peer Review, 8(2), $23-27$.

Tosey, P. (2006). Interfering with interference. In N. Jackson, M. Oliver, M. Shaw, \& J. Wisdom (Eds.), Developing creativity in higher education: An imaginative curriculum (pp. 29-42).New York: Routledge.

T. Zimmerer, N. Scarborough 2008 Essentials of Entrepreneruship and Small Business Management Upper Saddle River: NJ: Prentice-Hall. 